\title{
Teaching stellar interferometry with polymer optical fibers
}

\author{
M. Illarramendi, L. Arregui, J. Zubia, R. Hueso, A. \\ Sanchez-Lavega
}

M. A. Illarramendi, L. Arregui, J. Zubia, R. Hueso, A. Sanchez-Lavega, "Teaching stellar interferometry with polymer optical fibers," Proc. SPIE 10452, 14th Conference on Education and Training in Optics and Photonics: ETOP 2017, 1045216 (16 August 2017); doi: 10.1117/12.2266542

SPIE Event: 14th Conference on Education and Training in Optics and Photonics, ETOP 2017, 2017, Hangzhou, China 


\title{
Teaching "Stellar Interferometry" with polymer optical fibers
}

\author{
M.A. Illarramendi ${ }^{a}$, L. Arregui ${ }^{b},{ }^{\prime}$. Zubia ${ }^{c}$, R. Hueso ${ }^{a}$, and A. Sanchez-Lavega ${ }^{a}$ \\ ${ }^{\mathrm{a}}$ Applied Physics Department I, ${ }^{\mathrm{c}}$ Communications Engineering Department, \\ Faculty of Engineering. University of the Basque Country UPV/EHU. \\ Plaza Torres Quevedo 148013 Bilbao (Spain). \\ ${ }^{\mathrm{b}}$ Fundación Tekniker, Iñaki Goenaga 5, 20600 Eibar (Spain).
}

\begin{abstract}
In this manuscript we show the design of a simple experiment that reproduces the operation of the Michelson stellar interferometer by using step-index polymer optical fibers. The emission of stellar sources, single or binary stars, has been simulated by the laser light emerging from the output surface of the 2 meter-long polymer optical fiber. This light has an emission pattern that is similar to the emission pattern of stellar sources-circular, uniform, spatially incoherent, and quasi-monochromatic. Light coming from the fiber end faces passes through two identical pinholes located on a lid covering the objective of a small telescope, thus producing interference. Interference fringes have been acquired using a camera that is coupled to a telescope. The experiments have been carried out both outdoors in the daytime and indoors. By measuring the fringe visibilities, we have determined the size of our artificial stellar sources and the distance between them, when placing them at distances of $54 \mathrm{~m}$ from the telescope in the indoor measurements and of $75 \mathrm{~m}$ in the outdoor ones.
\end{abstract}

Keywords: Michelson stellar interferometer, polymer optical fiber, spatial coherence, fringe visibility, image processing

\section{INTRODUCTION}

Interferometry is nowadays a valuable tool in the study of stellar evolution as it allows the measurement of the basic parameters of stars with very high resolution. Teaching stellar interferometry in a practical way to undergraduate physics students and graduate students is not easy and it has hardly been developed, not even in specific areas such as optics and astronomy. This subject is largely ignored in most astronomy textbooks [1] and only the classic Michelson interferometer is briefly acknowledged [2]. On the other hand, although the Michelson stellar interferometer for determining the angular dimensions of astronomical objects is described in classic textbooks of optics [3,4], little has been written concerning experimental activities related to the optical principles of that type of interferometer, with the exception of some few works [5-8]. It is worth mentioning the experiment for undergraduate students reported by J.P. Sharpe and D.A. Collins [7], where the effect of the source size on optical spatial coherence is analyzed. However, these types of experiments have the weakness of lacking of a straight connection with the courses related to stellar studies. For instance, in such experiments, telescopes are not used or the light source is placed at short distances. In order to overcome those difficulties, and for the sake of introducing the subject in a practical way, we have developed an experimental method that allows direct characterization of distant extended sources, which can be performed both outdoors in the daytime and indoors. The experiment is based on the optical principles of the Michelson stellar interferometer, it needs simple equipment that is available in most astronomy and optics departments, and it can be integrated into existing courses with close interaction between theory and experiment. It has been developed as a laboratory exercise in the subject Space Interferometry within the Master of Science and Space Technology organized in the "Aula Espazio Gela" of the Faculty of Engineering of Bilbao [9-11]. The laboratory exercise is suitable for graduate or master courses on astronomy, optics or space science.

14th Conference on Education and Training in Optics and Photonics: ETOP 2017, edited by Xu Liu,

Xi-Cheng Zhang, Proc. of SPIE Vol. 10452, 1045216 • ( 2017 ICO, IEEE, OSA, SPIE

CCC code: $0277-786 X / 17 / \$ 18 \cdot$ doi: $10.1117 / 12.2266542$ 


\section{BASIC PRINCIPLES}

An optical stellar interferometer consists of two or more telescopes which detect light emitted from a distant system with the aim of obtaining information of the system with very high angular resolution. A simplified operation of this interferometer can be carried out by using a single telescope whose aperture is covered by a lid with two circular pinholes of variable separation between them. The optical fundamentals of this device are based on those of the Young's double-slit experiment. In this case, the beams emerging from each pinhole form interference fringes in the focal plane of the telescope. The quality of the fringes can be described quantitatively in terms of the fringe visibility $V$, which is defined as

$$
V=\frac{I_{\max }-I_{\min }}{I_{\max }+I_{\min }}
$$

where $I_{\max }$ and $I_{\min }$ are, respectively, the maximum and minimum irradiances of the interference pattern.

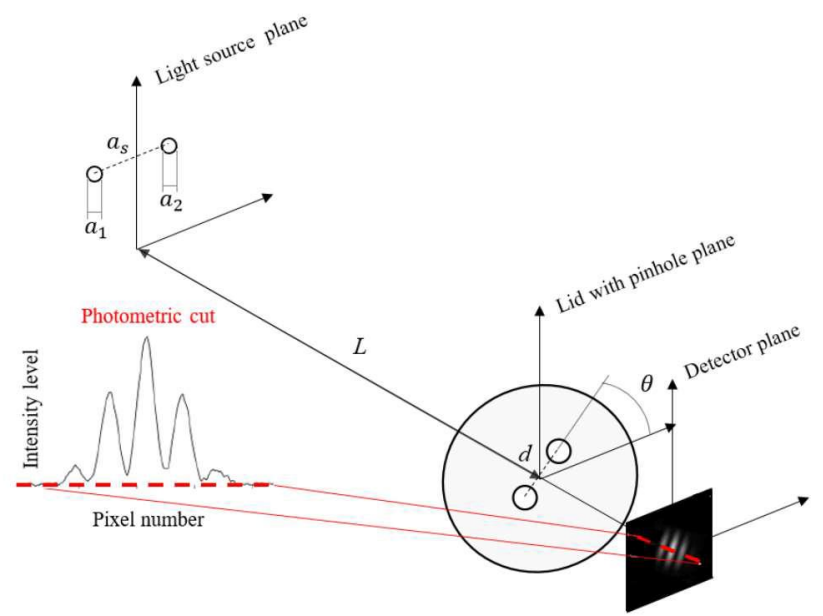

Figure1. Diagram showing the light source, the lid with pinholes and the telescope's detector.

From the theory of optical coherence $[3,12]$, the fringe visibility is interpreted as a measure of the degree of coherence in the two-pinhole system considering a quasi-monochromatic light coming from a spatially incoherent source. Particularly, the van Cittert-Zernike theorem describes the relationship between the irradiance distribution of the source and the corresponding fringe visibility. If the light source is described as a circular and uniform disk, which is the simplest model to describe the emission of a star, the fringe visibility is given by the following well-known expression [3]:

$$
V=2\left|\frac{J_{1}\left(\frac{\pi d a}{L \lambda}\right)}{\left(\frac{\pi d a}{L \lambda}\right)}\right|
$$

where $J_{1}$ is the first-order Bessel function of the first kind, $a$ the linear diameter of the source, $L$ the distance between the source and the two- pinhole system, $d$ the distance between the two pinholes (or baseline) and $\lambda$ the light wavelength. According to Eq. (2), the visibility $V$ decreases steadily from a value of 1 , when $\pi a d / L \lambda=0$, to a value of 0 , or total disappearance of the fringe pattern, when $\pi a d / L \lambda=1.22 \pi$. If the light source consists of two circular and uniform disks (1 and 2) separated by a certain distance (simulation of a binary stellar system), the corresponding fringe visibility is expressed as follows [13]: 


$$
V=\left|\frac{V_{1}^{2}+V_{2}^{2} f^{2}+2 f\left|V_{1}\right|\left|V_{2}\right| \cos \left(\frac{2 \pi d a_{s} \cos \theta}{L \lambda}\right)}{(1+f)^{2}}\right|^{\frac{1}{2}}
$$

Here $V_{1}$ and $V_{2}$ are the visibility functions for each disk given in Eq. (2), $f$ the total integrated flux received from source 2 at a given wavelength, to that received from source 1 , which is called the contrast of the binary system, $a_{\mathrm{s}}$ is the linear distance between disks, and $\theta$ is the angle between the imaginary line that joins the centers of the two disks with respect to the line that joins the two pinholes in the telescope (see Fig. 1). If both disks have the same linear diameter $\left(V_{l}=V_{2}=V\right)$, if their orientation is $\theta=0$ and if they emit the same radiant flux $(f=1)$, Eq. (3) can be simplified to the following expression:

$$
V=\left|\frac{J_{1}\left(\frac{\pi d a}{L \lambda}\right)}{\left(\frac{\pi d a}{L \lambda}\right)}\right| 1+\left.2 \cos \left(\frac{2 \pi d a_{s}}{L \lambda}\right)\right|^{\frac{1}{2}}
$$

This expression can be interpreted as a modulation of the visibility curve generated by a single disk, due to the cosine function produced by the binary system. It can be easily concluded that $V$ is zero either if $\pi a_{s} d /(L \lambda)=(n+1) \pi / 2$ $(n=0,1,2 .$.$) in the cosine function or if \pi a d /(L \lambda)=1.22 \pi$ in the first factor, corresponding to a single disk. The minima of $V$ due to the cosine function only drop to 0 if the contrast $f$ is 1 . The amplitude of the modulation decreases as $f$ is reduced.

If the distance $L$ and the light wavelength $\lambda$ are known, we can determine the diameter of a single disk by measuring the curve of the visibility $V$ of the fringes as a function of the baseline $d$ and then fitting Eq. (2) to this curve. Similarly, we can calculate the linear diameters of two disks together with their linear separation $a_{s}$ by measuring the dependence of the visibility $V$ of the binary system on $d$ and then fitting Eq. (3) to the curve. Although the aforementioned procedures are the most accurate ones, in some cases there are easier alternative procedures to determine the parameters of the light source. For the case of a single disk, the linear diameter of the source can be estimated by measuring the lowest value of $d$ at which the interference fringes disappear, in which case $d=1.22 \lambda L / a$. For a binary system in which the two disks have the same linear diameter with $\theta=0$ and $f=1$, the linear separation $a_{s}$ could be estimated by measuring the lowest value of $d$ at which the interference fringes vanish, in which case $d=\lambda L /\left(2 a_{s}\right)$.

\section{EXPERIMENTAL SET-UP AND PROCEDURE}

The indoor measurements were carried out in low-light conditions in a corridor located in the basement of our institution, while the outdoor ones were done by placing the source inside a room on the roof of a building of our school and the telescope in a laboratory on the top floor of another building. Figure 2 shows a scheme and a picture of the optical system designed for simulating stars. The light coming from a laser is split in two beams by means of a beam splitter (BS). These beams are launched into two step-index polymer optical fibers (SI-POFs) by means of SMA connectors (see Fig. 2). Finally, the free fiber ends are inserted into two $1 \mathrm{~mm}$-diameter holes located in an L-shape element, named positioner (PS). Specifically, they are inserted into the back of the PS up to the position where their end faces reach the front face of this element. The PS contains three pairs of holes that are horizontally aligned, with separations of 2, 3 and $4 \mathrm{~mm}$, respectively, from center to center (see Fig. 3). SI POFs have a large core diameter $(\sim 1 \mathrm{~mm})$ and a high numerical aperture $(\sim 0.5)$ or large-angle emission cone. The length of our fibers is $2 \mathrm{~m}$, which is enough to consider the free fiber end faces as uniform and spatially incoherent circular light sources [14], thus allowing us to use Eqs. (2), (3) and (4). The light-source system has been designed so that its parameters can be changed in a fast and easy way. On the one hand, the use of SMA connectors allows us to change the size of the source without difficulty, just by connecting SI POFs of 
different diameters. On the other hand, as can be seen in Fig. 3, the distance between sources can be changed just by inserting the fiber end faces in a different pair of holes in the L-shape element. In the outdoor measurements a fiber-beam expander is used to increase the size of the circular source and, at the same time, decrease the numerical aperture of the output light.
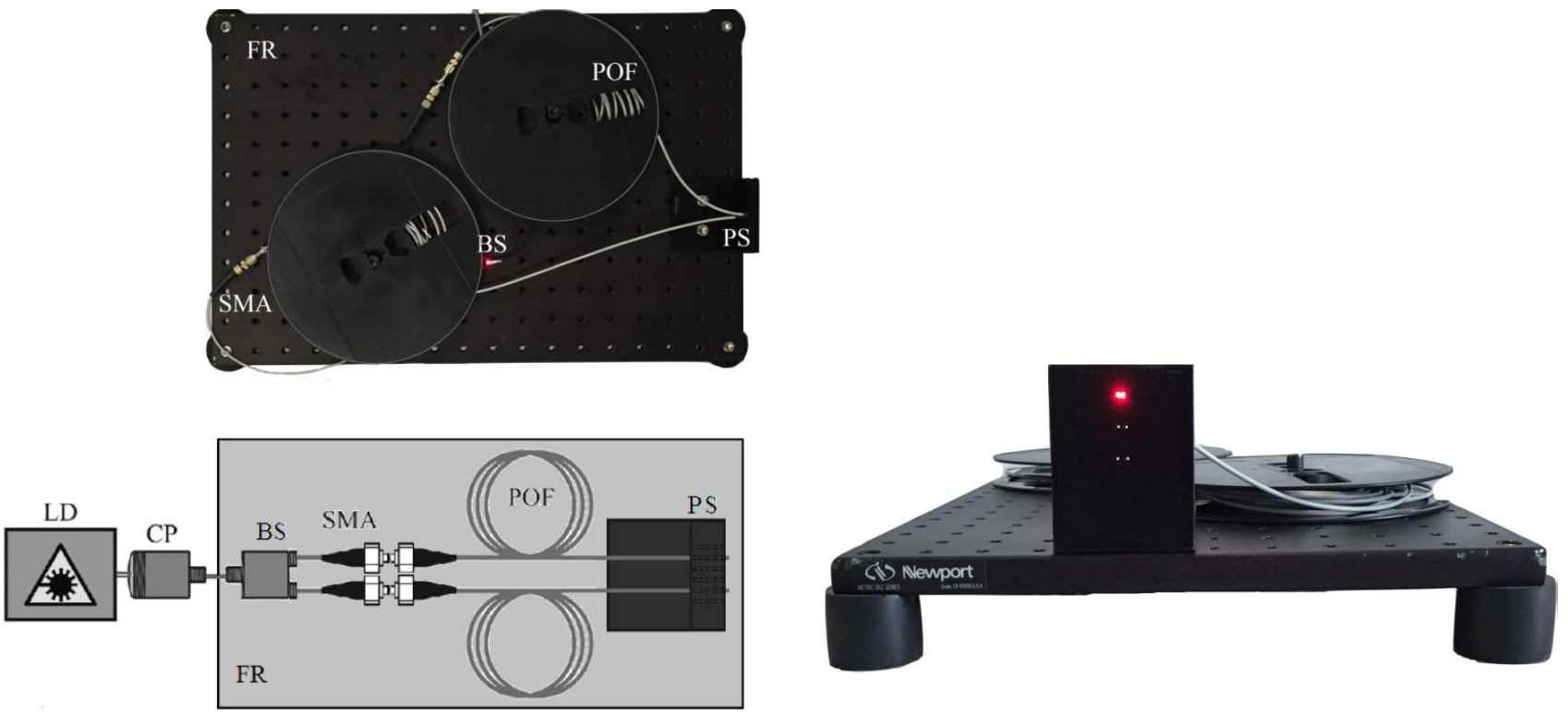

Figure 2. Top picture and scheme of the light-source system. LD: laser; CP: fiber coupler; BS: beam splitter; SMA connector; POF: polymer optical fiber; PS: positioner.

Figure 3. Front view of our artificial binary-star system with the fibers inserted in the L-shape element PS.

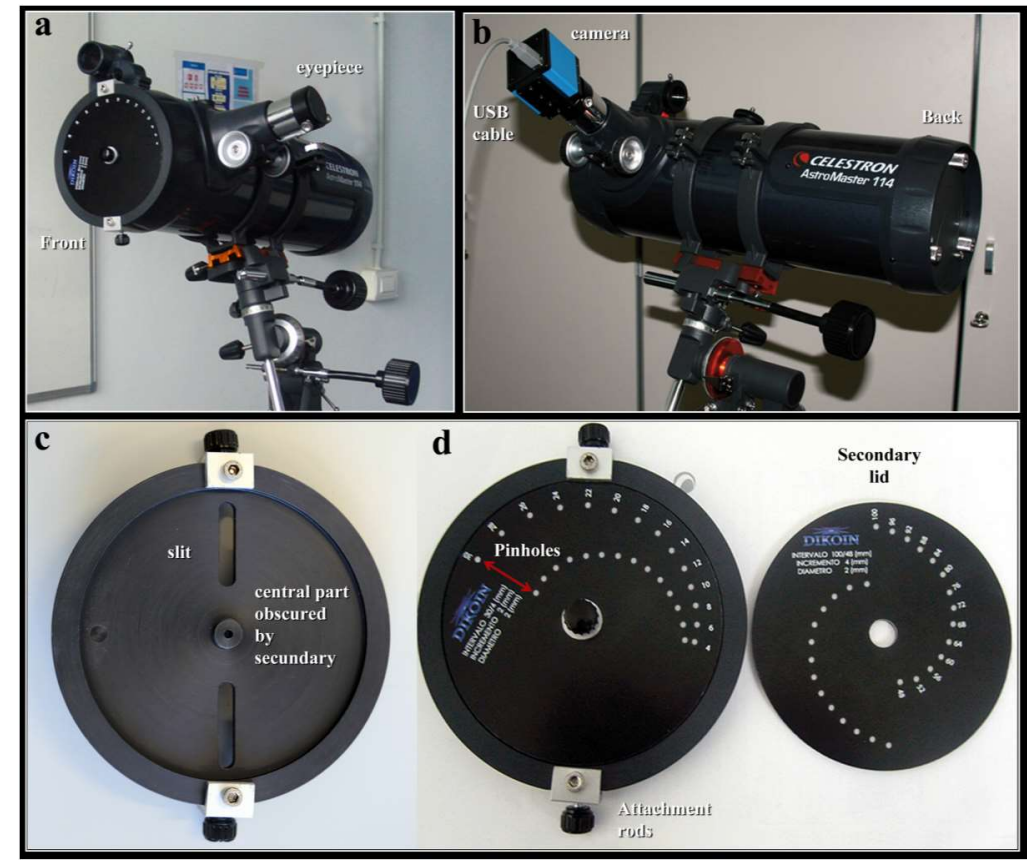

Figure 4. (a) reflector telescope with the lid; (b) telescope with the coupled camera; (c) internal slit; (d) external revolving lids with circular apertures. Separation distances between the pinholes are indicated on the mask in millimeters; the distance is changed by turning the revolving lid. 
Figure 4 shows the telescope used for the detection of the images (AstroMaster 114 EQ Newtonian, aperture $D=114 \mathrm{~mm}$ and focal distance $1000 \mathrm{~mm}$ ). Once the telescope was well focused, its aperture was blocked by a pair of identical pinholes separated different distances (Fig 4. (a)). Although there are various pairs of pinholes available on the same lid, an internal slit allows light to pass only through a pair of pinholes at a certain time, so different separations between pinholes can be attained by rotating the lid (Fig. 4(c)). Each pinhole has a diameter of $2 \mathrm{~mm}$. By using two different lids, the distances between the pairs of pinholes can be varied from $4 \mathrm{~mm}$ to $100 \mathrm{~mm}$ (Fig. 4(d)). The distance between the light-source system and the telescope was $L=54 \pm 1 \mathrm{~m}$ for the indoor measurements and $75 \pm 3 \mathrm{~m}$ for the outdoor ones. The fringe pattern can be visually observed through an eyepiece, and the digital images can be acquired with a camera located at the focal plane of the telescope (see Fig. 4(b)). For the detection, a DMK41AU02 camera equipped with the Sony ICX205AL CCD chipset was used [15]. This camera has a sensitivity of 0.05 lx and a dynamic range of $36 \mathrm{~dB}$. The pixel size is $4.65 \mu \mathrm{m}$, which renders high-resolution images. The outdoor experiments were carried on cloudy days in order to avoid the incoming of stray light onto the telescope, which diminishes the visual contrast and increases the noise in the experiment. Besides, the light of the room where the source was placed was switched off so as to ensure that low levels of light from the area surrounding the fiber end affect the experiment. In both cases, long exposure times (from $7 \mathrm{~s}$ to $10 \mathrm{~s}$ for indoor measurements and up to 2 minutes for outdoor ones) were needed in order to have an appropriate signal-to-noise ratio The values of the visibility in the acquired images were calculated by means of a program implemented in Matlab, but any other image-analysis software could have been used (for example, ImageJ [15]). $I_{\max }$ corresponds to the irradiance of the central maximum and $I_{\min }$ to the average of the two adjacent minima (see Fig. 5). All irradiance measurements were corrected for the background irradiance, which was estimated by averaging all the irradiance values outside the fringes.

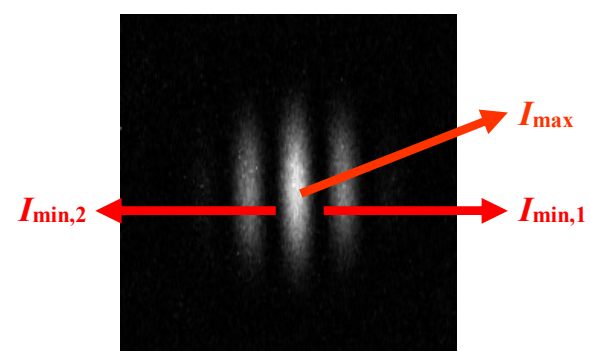

Figure 5. Interference pattern: central maximum and the two adjacent minima

\section{RESULTS}

The left graph of Fig. 6 shows the indoor measured fringe visibility produced by a single fiber as a function of the pinhole separation, for three different fiber diameters: $500 \mu \mathrm{m}, 750 \mu \mathrm{m}$ and $1000 \mu \mathrm{m}$ (nominal values). As can be seen in the graph, as the fiber diameter increases, the visibility decreases faster and the first minimum of the visibility is shifted to smaller baselines. A fit of Eq. (2) to the experimental points of each fiber, with $L=54 \mathrm{~m}$ and $\lambda=650 \mathrm{~nm}$, leads to the following results: $a=(510 \pm 30) \mu \mathrm{m}, a=(820 \pm 30) \mu \mathrm{m}$ and $a=(1130 \pm 40) \mu \mathrm{m}$ for each fiber, which differ by $3 \%, 10 \%$ and $13 \%$ from their nominal values respectively. The right graph of Fig. 6 shows the outdoor measured visibility curve produced by a single fiber. In this case the diameter of the source is $3 \mathrm{~mm}$. Notice that the error bars of the outdoor measured visibility values are larger than those of the indoor measured values. Fitting Eq. (2), in this case with $L=75 \mathrm{~m}$ and $\lambda=635 \mathrm{~nm}$, to the measured visibility data, we deduce a source diameter of $a=3.1 \pm 0.2 \mathrm{~mm}$. This diameter differs by $3 \%$ from the actual value.

Figure 7 shows the indoor measured fringe visibility produced by two fibers of the same diameter, namely $1000 \mu \mathrm{m}$, oriented parallel to the two pinholes of the lid of the telescope $(\theta=0)$ and emitting with the contrast $f=1$. Three separation distances between fibers were used: $2 \mathrm{~mm}, 3 \mathrm{~mm}$ and $4 \mathrm{~mm}$. As can be seen in the figure, the fringe-visibility curve produced by two fibers is modulated by the visibility curve created by a single fiber. As the distance between fibers as increases, the first minimum of the visibility occurs at smaller baselines, and the separations between consecutive minima are shortened. By fitting Eq. (4) to the experimental points with $L=54 \mathrm{~m}, \lambda=650 \mathrm{~nm}, f=1$ and $\theta=0$, we estimate, for each case, the values of the diameters of the fibers together with their separation. The results 
obtained from the fittings are shown in Table I. As an example, we show in Fig. 8 the interference patterns and the relative brightness profiles of the images obtained at four different pinhole separations of $d=4 \mathrm{~mm}, d=6 \mathrm{~mm}$, $d=10 \mathrm{~mm}$, and $d=16 \mathrm{~mm}$. It can be noticed that the maxima of the visibility curves get closer to each other as the distance $d$ increases. A similar behavior occurs in the case of a single source.
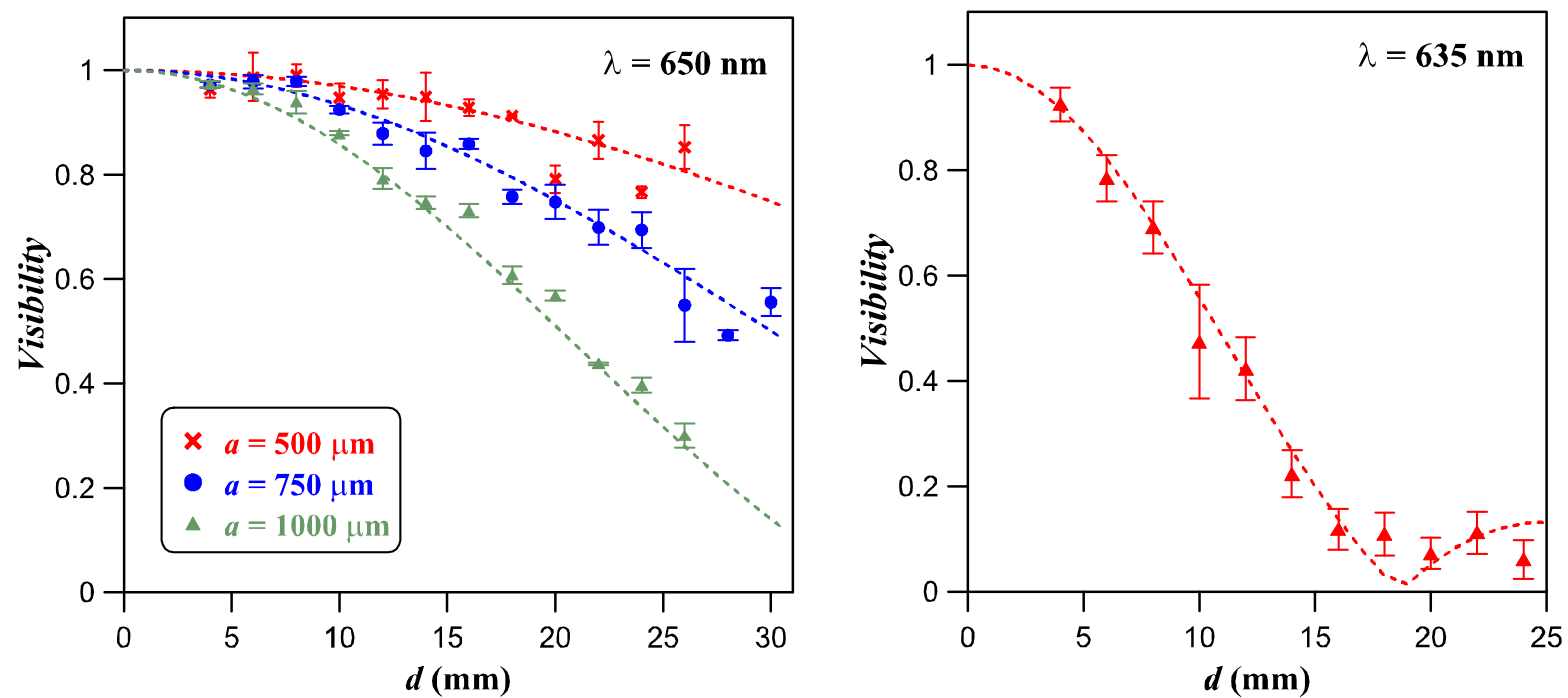

Figure 6. Visibility curves as a function of the pinhole separation. Left: indoor measurements. Right: outdoor measurement. The symbols show the measured visibilities obtained from the captured images. The dashed curves are the fits to the data using Eq. (2).
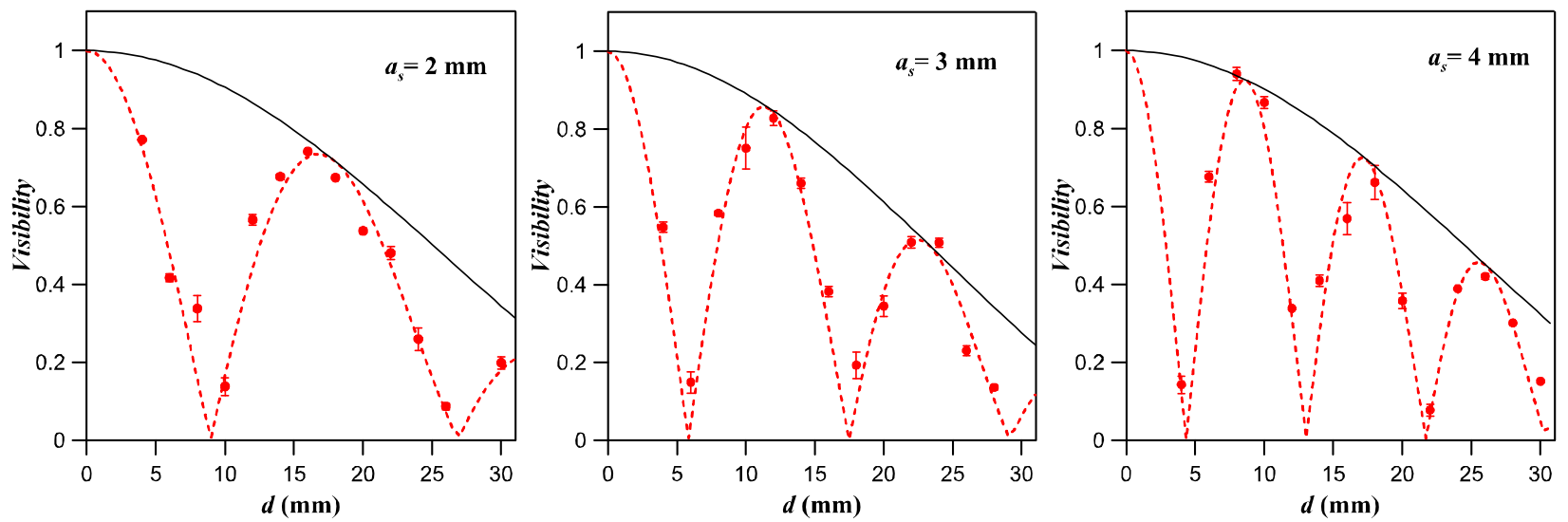

Figure 7. Visibility curve as a function of the pinhole separation obtained for binary circular sources of the same diameter $\mathrm{a}=1000 \mu \mathrm{m}$, with $f=1$ and $\theta=0$, separated by: $2 \mathrm{~mm}, 3 \mathrm{~mm}$ and $4 \mathrm{~mm}$. The solid circles are experimental points and the dashed lines are the fittings to the data using Eq.(4). The solid lines represent the curves corresponding to a single circular source.

Table I. Values of $a$ and $a_{\text {s }}$ obtained from the fittings of Eq. (4) to experimental points displayed in Fig. 7.

\begin{tabular}{|c|c|c|c|c|c|}
\hline Nominal & Fit & Error & Nominal & Fit & Error \\
\hline Size & Size & Size & Separation & Separation & Separation \\
\hline$a(\mu \mathrm{m})$ & $a(\mu \mathrm{m})$ & $\Delta a / a$ & $a_{s}(\mu \mathrm{m})$ & $a_{s}(\mu \mathrm{m})$ & $\Delta a_{s} / a_{s}$ \\
\hline $1000 \pm 1 \mu \mathrm{m}$ & $990 \pm 80 \mu \mathrm{m}$ & $2 \%$ & $2.0 \pm 0.1 \mu \mathrm{m}$ & $1.96 \pm 0.05 \mu \mathrm{m}$ & $2 \%$ \\
\hline $1000 \pm 1 \mu \mathrm{m}$ & $1060 \pm 70 \mu \mathrm{m}$ & $10 \%$ & $3.0 \pm 0.1 \mu \mathrm{m}$ & $3.02 \pm 0.07 \mu \mathrm{m}$ & $6 \%$ \\
\hline $1000 \pm 1 \mu \mathrm{m}$ & $1020 \pm 70 \mu \mathrm{m}$ & $5 \%$ & $4.0 \pm 0.1 \mu \mathrm{m}$ & $4.1 \pm 0.1 \mu \mathrm{m}$ & $2 \%$ \\
\hline
\end{tabular}



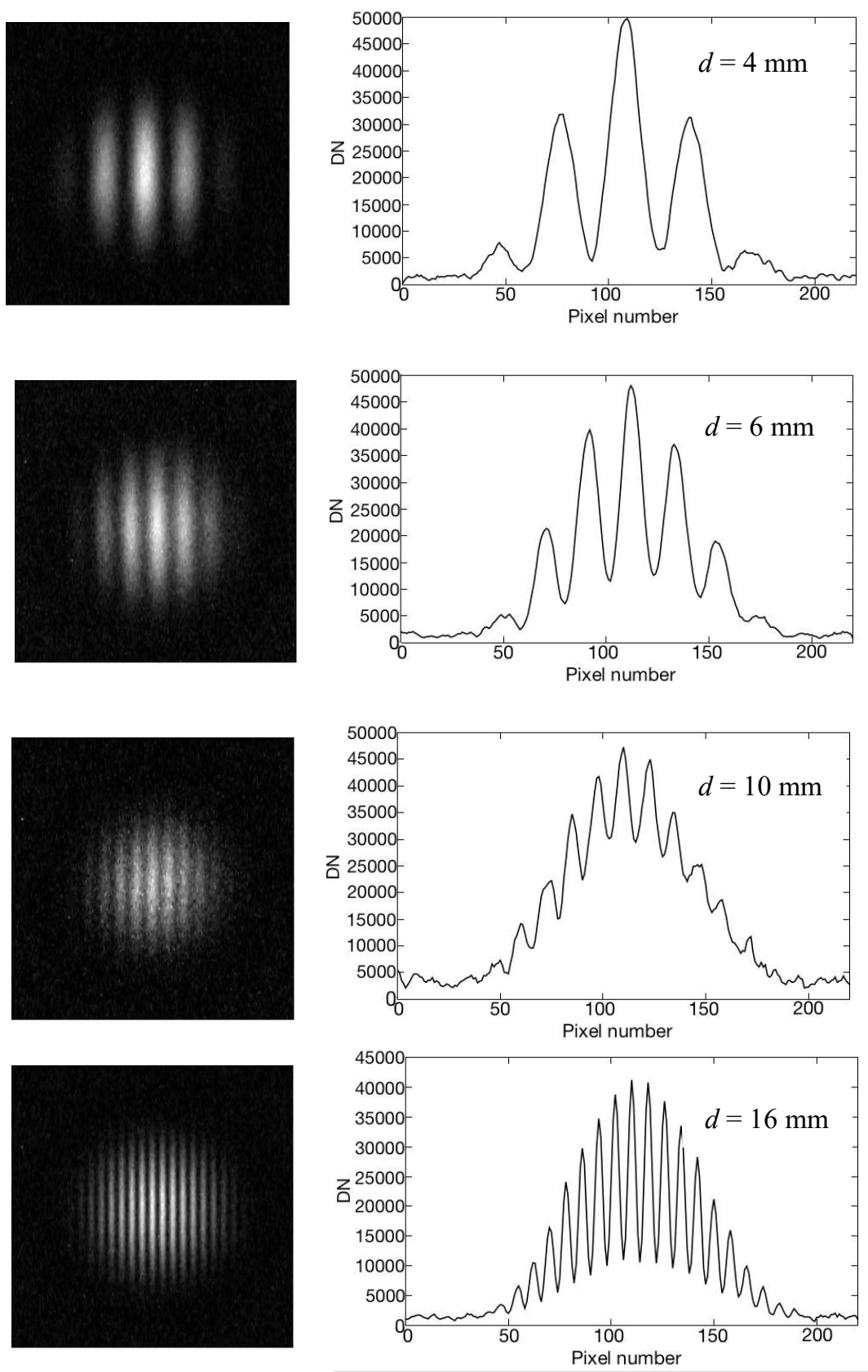

Figure 8. Interference patterns formed by light from a binary circular source placed at $L=54 \mathrm{~m}$ with three pinhole separations: $d=4 \mathrm{~mm}, d=6 \mathrm{~mm}, d=10 \mathrm{~mm}$, and $d=16 \mathrm{~mm}$. Light source: two fiber circular of $a=1000 \mathrm{~mm}$ with $a_{\mathrm{s}}=2 \mathrm{~mm}, f=1 \theta=0$, and $\lambda=650 \mathrm{~nm}$. The relative brightness profiles of the images are also shown for the same pinhole separations.

It should be noted that the $\mathrm{CCD}$ /telescope system could have been resolved the characteristics of ours sources by direct observation (without any lid). The limit of diffraction of the telescope is $1.4 "(1.22 \lambda / D)$ and the angular coverage of each CCD pixel is $0.95^{\prime \prime}$ and, in our experiments, we have come to resolve angular sizes of around $2^{\prime \prime}\left(0.00055^{\circ}\right)$. The angular resolution limit of our interferometer would be $0.6 "\left(\lambda / 2 d_{\max }\right)$, given that the maximum distance between the pinholes, $d_{\max }$, is smaller than the aperture of the telescope. Nevertheless, it must be taken into account that the objective of our demonstration is not to show how the interferometry can beat the Rayleigh resolution limit of the telescope but to demonstrate a simple operation of the stellar interferometer with single or double star systems. 


\section{SUMMARY}

We have designed an experiment set-up to demonstrate the fundamentals of the Michelson stellar interferometer. The emission pattern of stellar sources (single and double) have been reproduced by using $2 \mathrm{~m}$-long polymer optical fibers illuminated by a laser, which produce closely located circular, uniform, quasi-monochromatic and spatially incoherent emission patterns. The experiment can be performed both outdoors in the daytime and indoors. Using this experiment set-up, angular sizes of around 2" have been resolved with relative errors less than $16 \%$. The smallest size measured with our interferometer is comparable to Uranus (3.7") and Neptune (2.2") angular size. On the other hand, the experiment can also provide information about the relative orientation of the binary sources by taking into account an additional parameter, $\theta$. Furthermore, it is able to distinguish and deduce the contrast between the components of a binary system, such as the contrast of the binary system that recreates the conditions of the Alpha Centauri system with sources separated an angular distance of 15" [10].

The simplicity of the experimental device and the satisfactory results obtained make this experiment ideal to be implemented in postgraduate astrophysics, astronomy or optics subjects. On the one hand, the laboratory exercise implies the use of a telescope, a digital camera, and image processing tools. On the other hand, it provides the hands-on experience needed to understand the basic concepts of interferometry underlying the experiment. For introductory students, or only marginally addressing this subject, the experiment can be also carried out avoiding the image processing and the non-linear fitting, just finding the smallest value of the pinhole separation for which the interference fringes disappear. For advanced students coursing different subjects from optics to astronomy or image analysis, the experiment can be fully replicated as it has been carried out over the last years in our university.

\section{ACKNOWLEDGMENTS}

This work has been supported by the following institutions: Diputación Foral de Bizkaia/Bizkaiko Foru Aldundia through the Aula Espazio Gela, Ministerio de Economia y Competitividad under projects TEC2015-638263-C03-1-R and AYA2015-65041-P (MINECO/FEDER, UE), Gobierno Vasco/Eusko Jaurlaritza under projects IT933-16 and IT765-13 and ELKARTEK (KK-2016/0030 and KK-2016/0059) and by the University of the Basque Country (UPV/EHU) through programs UFI11/16 and UFI11/55.

\section{REFERENCES}

[1] Carroll B. W. and Ostlie, D. A. [An Introduction to Modern Astrophysics] Pearson Education, San Francisco, (2007).

[2] Karttunen H., Kroger P., Oja H., M. Poutanen M. and Donner, K. J. [Fundamental Astronomy], 5th ed. Springer-Verlag, Berlin, (2007).

[3] Born M. and Wolf, E. [Principles of Optics] Pergamon Press, Oxford, (1980).

[4] Hecht E. and Zajac, A. [Optics] Addison Wesley Publishing Company; 3rd edition (1997)

[5] Pryor M. J. "Measuring artificial star separation by interference" Am. J. Phys. 27 101-103 (1959)

[6] Ambrosini D., Schirripa Spagnolo G., Paoletti D. and Vicalvi, S. "High precision digital automated measurement of degree of coherence in the Thompson and Wolf experiment," Pure Appl. Opt. 7, 933-939 (1998).

[7] Sharpe J. P. and Collins, D. A. "Demonstration of optical spatial coherence using a variable width source" Am. J. Phys 79 554-557 (2011)

[8] Koda J., Barrett J., Shafto G. and Slechta, J. "A Michelson-type radio interferometer for university education" Am. J. Phys. 84 249-256 (2016)

[9] Illarramendi M. A., Hueso R., Zubia J., Aldabaldetreku G., Durana G., and Sanchez-Lavega A. “A daylight experiment for teaching stellar interferometry" Am. J. Phys. 82 649-653 (2014)

[10] Arregui L., Illarramendi M. A., Zubia J, Hueso R., and Sanchez-Lavega, A. "Interferometry of binary stars using polymer optical fibers" Eur. J. Phys., at press: https://doi.org/10.1088/1361-6404/aa7038 (2017)

[11] Master in Space Science and Technology http://www.ehu.es/aula-espazio/english/master.html

[12] Wolf E. [Introduction to the Theory of Coherence and Polarization of Light] 1st ed. Cambridge University press (2007) 
[13] Berger J. P. and Segransan, D. “An Introduction to Visibility Modeling”, New Astron. 51 576-582 (2007)

[14] Yoshimura H., Asakura T. and Takai, N. "Spatial coherence properties of light from optical fibers" Opt. Quantum Electron. 24 631-646 (1992)

[15] https://www.theimagingsource.com/

[16] ImageJ https://imagej.nih.gov/ij/ 